\title{
Navigation Doppler Lidar for autonomous ground, aerial, and space vehicles
}

\author{
Farzin Amzajerdian ${ }^{1}$, Glenn D. Hines ${ }^{1}$, Diego F. Pierrottet ${ }^{2}$, Bruce W. Barnes ${ }^{1}$, Larry B. Petway ${ }^{1}$, \\ and John M. Carson $\mathrm{III}^{3}$ \\ ${ }^{I}$ NASA Langley Research Center, Hampton, VA, USA 23681 \\ ${ }^{2}$ Coherent Applications Inc., Hampton, VA, USA \\ ${ }^{3}$ NASA Johnson Space Center, Houston, Texas, USA \\ f.amzajerdian@nasa.gov
}

\begin{abstract}
A Doppler lidar instrument has been developed and demonstrated for providing critical vector velocity and altitude/range data for autonomous precision navigation. Utilizing advanced component technologies, this lidar can be adapted to different types of vehicles. OCIS codes: (280.3640) Lidar; (120.2920) Homodyning; (060.2340) Fiber optics components.
\end{abstract}

\section{Introduction}

A coherent Doppler lidar has been developed to address NASA's need for a high-performance, compact, and costeffective velocity and altitude sensor onboard its landing vehicles. Future robotic and manned missions to solar system bodies require precise ground-relative velocity vector and altitude data to execute complex descent maneuvers and safe, soft landing at a pre-designated site. This lidar sensor, referred to as a Navigation Doppler Lidar (NDL), meets the required performance of the landing missions while complying with vehicle size, mass, and power constraints. Operating from over four kilometers altitude, the NDL obtains velocity and range precision measurements reaching 2 $\mathrm{cm} / \mathrm{sec}$ and 2 meters, respectively, dominated by the vehicle motion. Terrestrial vehicles may also benefit from NDL data products as enhancement or replacement to GPS systems, radars, and range imaging lidars, with some modification in its current configuration which is currently optimized for NASA landing applications.

\section{NDL System Description}

The principle of the lidar operation is illustrated in Fig. 1 showing the modulation waveform consisting of three segments: up-ramp chirp, constant frequency, and down-ramp chirp. The resultant returned waveform from the target is delayed by $t_{d}$, the light round trip time. When the target or the lidar platform is not stationary during the beam round trip time, the signal frequency will be also shifted up or down, depending on the velocity direction, due to the Doppler effect. When mixing the two waveforms at the detector, an interference signal is generated whose frequency is equal to the difference between the transmitted and received frequencies. In absence of velocity along the laser beam, the signal frequency during the "up-ramp" and "down-ramp" periods are equal and their magnitude is directly proportional to the distance to the target. When the vehicle is moving, the up-ramp and down-ramp frequencies will not be equal and their difference is related to the Doppler velocity. The target range and magnitude of the velocity component along the laser beam are determined through the following simple equations:

$$
R=\left(\frac{T C}{2 B}\right)\left(\frac{f_{I F}^{+}-f_{I F}^{-}}{2}\right) \quad V=\left(\frac{\lambda}{2}\right)\left(\frac{f_{I F}^{+}+f_{I F}^{-}}{2}\right)
$$

where $f_{I F}^{+}$and $f_{I F}^{-}$are the intermediate up-ramp and down-ramp frequencies, $B$ is the modulation bandwidth, $T$ is the waveform period, $C$ is the speed of light, and $\lambda$ is the laser wavelength. The constant frequency segment also produces a Doppler velocity that can be used for eliminating data dropouts when either up-ramp or down-ramp frequency is very close to zero, and allows for eliminating the measurement ambiguities that may arise in certain scenarios.

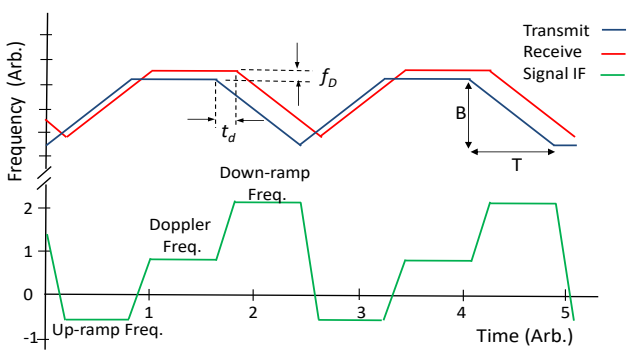

Figure 1. Linearly frequency modulated transmitted beam and returned signal, and the resulting intermediate frequency (IF) of the homodyne signal.

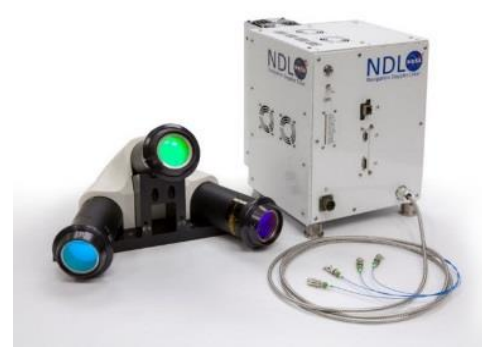

Figure 2. Prototype NDL demonstrated for space vehicles landing on planetary bodies. 
The NDL in its current configuration transmits three laser beams at different pointing angles toward the ground to measure range and velocity along each beam using a frequency modulated continuous wave (FMCW) technique. ${ }^{1}$ The three line-of-sight measurements are then combined in order to determine the three components of the vehicle velocity vector and its altitude relative to the ground. Figure 2 shows the NDL prototype system developed for a series of flight tests to demonstrate its capabilities and viability for future landing missions to planetary bodies. The prototype consists of an electronic chassis and an optical head. All the lidar components including the transmitter laser, receivers, and signal processor, are housed in the electronic chassis. The optical head consists of three transmit/receive lenses connected to the chassis via a long fiber optic cable.

\section{Operation and Performance}

The NDL performance and capabilities have been demonstrated through extensive ground tests, helicopter flight tests, and onboard autonomous rocket-powered test vehicles while operating in open and closed-loop with a guidance, navigation, and control (GN\&C) system. ${ }^{1,2}$ Global Positioning System (GPS) is commonly used in terrestrial navigation for vehicle position and velocity knowledge. In the absence of a GPS signal, past landing missions to planetary bodies relied on radar to provide the necessary data to execute descent and landing maneuvers. Radar limitations however, place unnecessary constraints on future landing vehicles adding risk to ambitious mission objectives. For this reason, we have developed and demonstrated the Navigation Doppler Lidar (NDL) as an alternative to radar sensors. The NDL offers several critical advantages compared to radar, including significantly higher precision with reduced size, weight, and power. In addition, the laser-based NDL sensor does not suffer from measurement perturbation from terrain features or signal ambiguity from transmitted side lobes, and is far less susceptible to signal clutter, such as returns from the lander structure or jettisoned vehicle components such as heatshields. The higher quality data provided by the NDL will enable both a more precise navigation towards the designated landing site and a well-controlled touchdown (with greater stability and lower impact loads). This translates into lower fuel reserve and smaller leg span for a landing vehicle and thus further reduces vehicle mass, therefore the NDL can potentially reduce the overall cost and risk of landing missions and enable new capabilities for planetary exploration missions including missions to the Moon, Mars, asteroids, and planetary moons.

The NDL can also benefit terrestrial applications such as aircraft navigation without reliance on external satellite signals. Conventional aircraft GN\&C systems combine Inertial Measurement Unit (IMU) data with the signals from a Global Positioning System (GPS) to determine the vehicle position and velocity vector. An IMU has an excellent response to rapid motion but suffers from an accumulated error over time (drift) while GPS can provide accurate longterm data. However, the GPS signal can be blocked or jammed by intentional or unintentional interference causing significant deviation in the navigation solution. Also, GPS position (altitude) data is referenced to sea-level and is of limited value when landing on a moving platform or in limited visibility conditions, or when autonomous navigation and landing is required. ${ }^{3} \mathrm{NDL}$ is particularly attractive for helicopters operating in brownout and other forms of Degraded Visual Environment (DVE) in which loss of visual ground reference occurs. Precise surface-relative position and velocity data are critical for implementing a reliable "see-and-remember" capability in which the pilot is provided with a synthetic visual display.

A modification of the NDL can also make a dramatic impact to the ongoing efforts for achieving safe and efficient autonomous ground vehicles. By scanning its laser beam, the NDL can provide 3-D images of the surrounding plus the velocity of moving objects within its field of regard. Fully autonomous cars can only become feasible when the onboard sensors can replace all the required sensing functions of a human driver. ${ }^{4}$ This includes distance, and relative speed and direction of other cars and people. The driverless car demonstration tests to date have been based on using a laser sensor with a scanning mirror to provide a static 3-D view in the vicinity of the car. However, such sensors cannot directly or accurately provide the critical speed and direction of moving targets. On the other hand, the NDL can provide both 3-D range and Doppler (velocity) images of surroundings.

\section{References}

[1] F. Amzajerdian, D. F. Pierrottet, G. D. Hines, L. B. Petway, B. W. Barnes, J. M. Carson III, "Development and Demonstration of Navigation Doppler Lidar for Future Landing Mission,“ AIAA Space Forum, 10.2514/6.2016-5590 (2016)

[2] V. E. Roback, D. F. Pierrottet, F. Amzajerdian, B. W. Barnes, G. D. Hines, L. B. Petway, P. F Brewster, K. S. Kempton, and A. E. Bulyshev, "Lidar sensor performance in closed-loop flight testing of the Morpheus rocket-propelled lander to a lunar-like hazard field," Proc. of AIAA Science and Technology Forum and Exposition (2015)

[3] Tim. Waanders, T. Münsterer, M. Kress, “Sensor supported pilot assistance for helicopter flight in DVE,” Proc. SPIE 8737 (2013)

[4] Jacques Cochard, Clémentine Bouyé, "From advanced driver assistance to autonomous driving: perspectives for photonics sensors," SPIE 9754 (2016) 